\title{
3. Some ethical imperatives for the computing profession ${ }^{1}$
}

\author{
Neville Holmes
}

\section{Introduction}

This chapter focuses on the ethical responsibilities of the computing profession. Governance should be based on ethics and be imposed by authority. The ethical aspects of digital technology should, therefore, be understood before its governance can be expected to be effective. Also, to be respected, governance of the industry should be administered by a knowledgeable authority; that is, by a professional body encompassing the entirety of digital technology. This technology has been developed and exploited in a quagmire of commercialism and hyperbole in which intellectual property law and market dominance determine the direction of development much more than the nature of the technology available and capability of the technologists involved. For its ethics to be understood and its governance to be properly designed, a very broad viewpoint must be taken of digital technology. This essay is intended to take such a viewpoint.

\section{Technology and ethics}

It is nowadays almost trite to say that our world is the way it is because of technology. Everything we do — watching, eating, listening, travelling — is embedded in technology, very often digital technology. Ethically, such a claim is grossly misleading and, in principle, completely false. In a fundamental way, technology can carry no direct responsibility for anything.

\section{Technology}

Technology is something people use. Consider the following extract from the entry for technology in an authoritative dictionary:

\footnotetext{
1 This essay expands on my presentation to the EtGovICT2008 symposium, Canberra, 1-2 May 2008. It draws on essays that I have written since 2000 for a monthly column, 'The profession', which appeared for more than 10 years in Computer, the house journal of the IEEE Computer Society $(<$ http://www.computer. org $>$ ). For educational or research purposes only, these essays are archived at $<$ http://tinyurl.com/wnhrkyv $>$
} 
Professionalism in the Information and Communication Technology Industry
a. A discourse or treatise on an art or arts; the scientific study of the practical or industrial arts
b. Practical arts collectively
c. A particular practical or industrial art
Oxford English Dictionary (OED) (2nd edition)

In a technological context, art has a personal meaning:

I. Skill; its display or application

$O E D$

Thus, technology is, by definition, personal because skill is personal. Technology is practiced by technologists and applied industrially by engineers, though the distinction between the two is usually blurred. Products of technology are used by other people who are, thus, the indirect users of technology. People decide what technology is used for and must, therefore, take responsibility for the effects of such use.

\section{Ethics}

Responsibility might bring blame or praise, or something in between. The nature of this spectrum is the subject matter of ethics, that is, of

II. 2. The science of morals; the department of study concerned with the principles of human duty

4. In a wider sense: The whole field of moral science, including besides Ethics properly so called, the science of law whether civil, political, or international

$O E D$

The adjective moral is defined as

a. Of or pertaining to character or disposition considered as good or bad, virtuous or vicious, of or pertaining to the distinction between right and wrong, or good and evil, in relation to the actions, volitions, or character of responsible beings; ethical

$O E D$

The nub here is that morality, the focus of ethics, has to do with the entirely personal ideas of right and wrong, of good and evil. These ideas cannot be 
applied to technology, or to the machines or processes proceeding from the use of technology. Only users of technology, be they direct or indirect users, can be held responsible for any good or evil resulting from its use.

\section{Anthropopathism}

The problem with being responsible for evil or wrongdoing is the human desire to avoid the responsibility, at least in people with a normal social sensitivity. The complexity of technology seems to provide an excuse for a bad outcome arising out of its use. The driver of a car causing an accident might seek to blame the car itself for its imputed malfunction, or the other driver, or the road conditions. Malfunction might well be the fault of the manufacturer or designer or maintainer. The other driver might well be responsible. For all that, there is usually some level of responsibility that must be borne by the driver at fault.

With computers, it's a bit different. There isn't another driver, and the software and hardware manufacturers use complexity to avoid blame for any but the most blatant fault. So, the computer, its software, or both, get the blame for malfunction. When a business or government department using a complex computing system blunders, blaming the system seems to be standard practice. There is a distinction to be remembered between cause and responsibility.

The greatest social role of the computer is as a scapegoat. This possibility was foreseen by computing pioneers many decades ago. In developing an international standard vocabulary for the profession, they put the most important terms first, data and information, ${ }^{2}$ and deliberately designed their definitions to bring out the contrast between people and technology.

Data. A representation of facts or ideas in a formalized manner capable of being communicated or manipulated by some process.

Information. In automatic data processing the meaning that a human assigns to data by means of the known conventions used in its representation.

\section{IFIP-ICC Vocabulary of Information Processing 1966}

The distinction can be more simply made by saying that 'only people can process information; machines can only process data'.

The computing profession has a moral responsibility to discourage the use of anthropopathic language when discussing computers. One place to start would be with the adoption of the standard vocabulary, in particular with

2 These were originally first and third, with signal second, but are lately first and second. 
respect to the most important terms: data and information. When faced with this specific suggestion, computing people typically aver that it's far too late because the media and the public at large have come to accept the two terms as interchangeable. This is no excuse. If the profession promoted and practiced the distinction within the profession, it would gradually be adopted by others, starting with technical journalists. Indeed, the profession could press publishers to adopt the distinction in their style guides, and to enforce its use by their authors.

Anthropopathic language is rife in the computing industry, far in excess of this example. The next worst example, perhaps even more socially damaging, is the reckless use of intelligent in respect of machinery. There are many reasons to condemn this usage. Intelligence is essentially, and despite the misleading name of IQ tests, a collection of social skills. Machines have, in themselves, nothing at all to do with sociality, and it is grossly unethical to suggest that they do. The ugly initialism AI should be dropped, together with artificial intelligence, and replaced by neutral terminology; perhaps the old term algoristics could be revived. When faced with this suggestion, computing people typically say that, really, some programs do exhibit aspects of intelligence. This excuse misses the point. The computing profession has a moral responsibility to maintain a distinction between people and machines, and for this reason professionals should avoid any suggestion that machines possess intelligence, artificial or not.

\section{The computing profession}

Urging the computing profession to take a stand on ethical issues would be all very well if the profession were well defined, generally respected, and widely recognised. Sadly, it is not. Until 20 years or so ago, the profession was in good shape. Digital computers were costly and used almost entirely within large organisations that employed well-trained people to run them. These people had the straightforward tasks of writing programs, either to process numbers and text in bulk in the case of business data processing, or to carry out complex numeric calculations in the case of scientific data processing. Computer societies were set up to promote professional recognition for workers in computing and these grew rapidly. So did tertiary courses in computer science, of academic orientation, and information systems, of business orientation. A growing proportion of people working with computers had these tertiary qualifications and saw fit to join their local computer societies.

When computers became cheap enough to be used by small enterprises, or by small groups within large enterprises, however, fewer of their users saw themselves as computing professionals requiring tertiary qualifications to 
practice their profession. Computer society memberships levelled off. When computers became cheap enough to be owned personally, they proliferated, their coupling over the Internet became common, and the population of computer users grew rapidly. Increasing use of digital chips in everyday devices made many more people computer users.

That computer society memberships and tertiary student enrolments have declined recently implies that the professional societies are not in a position to apply governance to the generality of workers in the computing industry, and that the very idea of professional computing needs revision. To get some idea of what revision might be appropriate, the role of digital technology in society needs to be examined.

\section{Digital technology}

Digital technology pervades our society, but then, from an overall point of view, it always has. Our social development has only been made possible by the development of digital technology. Understanding this depends on appreciating the basic nature of digital technology, and its contrast to analogue technology.

Digit. 3. a. Each of the numerals below ten ... expressed in the Arabic notation by one figure

Digital. 4. Of, or pertaining to, or using digits; spec. applied to a computer which operates on data in the form of digits or similar discrete elements

$O E D$

Digital technology is based on being able to discriminate between a few discrete elements. Analogue technology is not. The abacus is a digital calculator; the slide rule is an analogue calculator.

Early telegraphy used digital technology to allow semaphores and Morse signals to reliably transmit messages over long distances because the encoding was limited to only a few discrete elements. More recently, telephony was an analogue technology transmitting an electrical signal, analogous to the original acoustic waveform, and thus allowing it to be regenerated, providing too much noise did not accumulate during the often many stages of transmission.

The impressive success of modern digital technology springs from its binary encoding since, having only the two distinct elements - conventionally named zero and one - allows large amounts of noise to be cleaned off a signal at every stage. Binary encoding also allows systematic redundancy to be added when 
heavy noise is expected so that the received binary signal can be corrected. More complex binary encodings, such as alphabets, are represented as binary digit (bit) combinations.

\section{Language}

The basis of human society is language, originally only spoken or sung. Vocal language depends on distinguishing between words common to the vocabularies of the speaker and the listener. To distinguish between words is relatively easy, despite the large number of different words in a typical vocabulary, because spoken words are encoded from a limited number of phonemes. In English, the word hit has three phonemes, hint four, the second word being distinguished by the extra phoneme. Spoken language is thus a digital technology, a skill to be displayed and applied by its users. Speech, or an equivalent such as signed language, is a skill necessary to the functioning of a complex society. While speech is built from a digital vocabulary, it also transmits non-digital information to a listener, information such as the identity and mood of the speaker. Such extra information is the lifeblood of professional acting and political oratory.

Speech is transient. Written language was developed as a durable representation of speech to allow tyrants and bureaucrats to control people over a widespread area. More recently, written language has in many countries been made available to the general populace. Such languages are digital in being represented by combinations of relatively few discrete elements. There are many writing systems, though there are many more spoken languages. The elements of alphabetic systems, for example, in principle represent phonemes of the spoken language, though extra elements such as the decimal digits represent words, and punctuation symbols suggest non-digital content. Written language used administratively ideally expresses rules unambiguously. If written language is used personally it needs to have the nuances of its meaning added by the reader. Reading is a thoughtful and imaginative activity, of richness and variety resulting from the mood and experience of the reader.

The digital computers of 50 years ago were used for administration and research. Business and government used them on transaction records, and scientists and engineers used them for numerical computation. As they became more capable, in particular when they grew beyond a character set consisting mostly of digits and upper case letters, they found further uses. In the 1980s, the personal computer and its printer began replacing the typewriter, a digital machine that had held great social significance. Connecting personal computers to the Internet added significant scope to the use of written language through usergroup and personal email messages and, later, through the World Wide Web of documents. 


\section{The computing profession}

Over the last 20 years, the development of binary digital machinery and communication has spread beyond traditional computing. The old telephonic cycle of speech being encoded, transmitted, and decoded by medium of an analogue electrical signal has been reversed. Analogue representations of material such as sounds and images are now being directly encoded digitally for storage and transmission, before being used to recreate the original sounds and images in a close approximation of their original analogue representation. While this has had a profound social effect, discussed below, it has also had a very significant professional effect. It has led to the use of computers being spread throughout the formal professions, even to the control of theatrical performance.

Though they are helped by relatively well-recognised software engineers, professionals of many kinds are using digital technology without using other computing professionals. And, in business and government, management prefers to use digital technology to drive employees through well-defined routines by deploying large, general-purpose software packages that have been built and installed by software engineers. Thus, the computing profession is faced with two problems.

The first problem is that the software engineering profession is becoming well recognised and accepted. Sooner or later it will become a formal branch of engineering, and it is arguable that this is only being delayed by the peculiar refusal to acknowledge the need to distinguish the trade of programming and to employ technicians skilled in that trade in the same way that other branches of engineering employ appropriate technicians. When this happens, the computing profession will have to adapt in some way to the loss of what many in the profession see as their most important component.

The second problem is that neither the personal computer user, nor the professional computer user, sees a need for computing professionals beyond software engineering. This is leading to a very significant reduction in tertiary computing enrolments. A factor here is that secondary education is diverting much of its focus to developing skills in the use of computers, a mistaken diversion in my opinion. Consequently, graduates from secondary school see neither a need for further education in computing nor a prospect for getting rewarding employment as a graduate in computing or information systems.

These problems argue for a revision of the computing profession by the professional body aiming to administer that profession. In my opinion, the way forward must be to recognise and promote the trade of programming. This would free up computing professionals to focus on the people using computers rather than the computers themselves. After all, it is said to be a 
characteristic of learned professions that they look primarily to the interests of people, and are responsible for how their technology benefits those people. Such is the ethical aim of a profession. However the computing profession is reformed, its professional body cannot prescribe ethical behaviour and enforce that prescription until its professional scope is clearly and restrictively defined. Reform is thus an ethical imperative.

\section{Computing and people}

The development of human societies has long depended on the use of language of one kind or another. Linguistic communication has made change possible in both human relationships and human activities. Socially and ethically, change in human relationships is of prime significance, and modern digital technology is having its most important effect on these relationships. To understand what is happening to our society, the role of language in past changes needs to be understood. While these changes have been both varied and complex, there do seem to be significant generalities, which are especially evident when looking at present-day society.

\section{Oral society}

In oral societies, social relationships were necessarily established by conversation. Cooperative activities depended on debate, and social hierarchies came from some people in a community having the authority to issue commands to others. In some languages the social structure is built into the vocabulary, for example, with different pronouns used for people of higher social status and people of lower. Of special significance are names for family relationships because they declare important responsibilities. The family is by far the most significant component in oral societies. Childhood is used by families to ensure that the next generation is capable of sustaining the social structure.

\section{Literate society}

Written language has been, for almost all of its history, confined to a privileged administrative stratum of society. This is most clearly seen in the role of the book as the main material source of authority in early religious society and in bureaucratic society. Quite recently, the digital technology of printing and the burgeoning of urban society made popular books affordable, widespread literacy desirable, and general education practical. This development created a discourse both social and technical at a level above the family and village. Without this discourse our modern society would not have happened. 
The development of technology of any kind depends on the accumulation of knowledge. To accumulate steadily, knowledge must be durably represented, examined widely, and extended incrementally. The context for the development both of technology and of prosperity is the enlargement of society. Going far beyond the farm, the village, and the town, thousands upon thousands of people must be able to live and work together.

There have been several factors underlying whatever success modern society may claim to have won. First, is the oral underpinning of social and occupational everyday life. This has been created by the society of the family followed by and supporting the society of the school. The further that education goes, the richer the social and occupational intercourse. Second, is the literacy and numeracy that underpins life in wider society, recreational life in associations and meetings, and vocational life in the workplace and office. Literacy and numeracy make such extraneous personal linkages possible. Third, is the widening of social contact fostered by the development of personal transport and communication. Interaction with a variety of people makes life interesting and develops sociality. Fourth, is the personal enrichment fostered by the enlargement of social and vocational life. A richer life is not only a matter of personal experience, but, perhaps more importantly, of self knowledge through contemplation and the need for social sensitivity.

\section{Binary digital society}

This review of the social background of modern society has an old-fashioned flavour, and would be probably be looked at askance by young people today. Does this invalidate it? Well, validity depends on ethical values, and these seem to have changed over recent years. I have become increasingly aware of this since I moved from a semirural location to an inner suburb of a regional city. Being able to walk to shop or dine out, or just for the pleasure of wandering through tree-lined streets, is a delight. But the delight is tempered by evidence of values that are alien and abhorrent to me.

A few examples, then. School students who have just bought a hamburger drop the paper bags at their feet; a rubbish bin that is located a couple of paces away remains unused. By late afternoon, the plaza outside the fast-food shop is littered with discarded food and wrappings. Footpaths and nature strips near shops and hotels are spread with shards of broken glass. The young driver of a loud, speeding car and his three young passengers give me a one-finger salute and shout obscenities at me when I look wonderingly in their direction as they corner in front of me, though their shouts are softer than the thumping in the car that passes for music. 
But these are just personal examples of what seems to me to be a drastic change in values and ethics from those that applied in my own youth. These are more evident in things I read about so frequently in the newspapers - violence, murders, pornography, rape, binge drinking, dangerous driving, robberies all too often being committed by adolescents. And internationally, .... Maybe the seeming prevalence of these distressing events is just a result of what the media chooses to publish, but this would be, in any case, a change in values. Maybe I'm just an old-fashioned dodderer with old-fashioned values. If so, then I think the old-fashioned values should be brought back.

My observations, however, suggest to me that the problem is deeper than that, and that society has been badly weakened by the use of modern binary digital technology. The tragically misnamed social media seem likely to continue this weakening, perhaps even accelerate it.

Binary technology now gives priority to sensation and perception. Old-fashioned literacy and numeracy gave priority to imagination and contemplation. Popular music is rhythmic to excite, old-time music is melodic to soothe and charm. Modern games involve the player in rampant conflict, old-time games like chess and bridge are social and thoughtful. Nowadays, idle time, which used to be spent reading a book or chatting, is spent in excited watching of a screen. In short, modern culture is based on the satisfaction of individual desires, whereas old-time culture was based on family and other group sociality.

A great many influences have effected this change, and most of these influences have some technological aspects. It could be argued, for example, that production technologies have made consumer goods cheaper and that cheaper transport has made them more varied. Then, popular media has promoted individual desires for the consumption of these goods. Such technologies aren't basically digital, but digital technology has played a significant part in their recent development, as well as greatly amplifying the variety of consumable items.

\section{The ethical imperative}

The goodness or badness of the dominance of consumerism in our modern society is an ethical judgement. The diminution of sociality, however, whatever its cause, cannot be considered a good thing. That the computing profession and its binary digital technology has a significant rôle in that diminution is certain, however uncertain the exact nature of that rôle may be. The profession, therefore, has an ethical imperative to work to reverse the diminution. Some possibilities are obvious. 


\section{Basic skills}

Consider in today's adolescent culture the four factors given above as underlying the success of modern society. First is the oral underpinning of everyday life, starting in the family. This is declining. A current problem in early schooling is that too many children starting school cannot converse. They understand spoken language but they cannot use it themselves. Second, is the literary and numerary underpinning of recreational and vocational life in wider society. Literacy and numeracy are both declining, at least in 'developed' societies like Australia. Both the vocabulary and the arithmetic of school leavers have, on average, become greatly reduced. Third, is the widening of social contact through better personal transport and communication. Some commentators consider that social contact among many young people is being narrowed by developments such as the prevalence of single-parent and working-parents families, by protective measures such as driving children to school and removing social activities like physical education from the curriculum, and by gluing students to Internet terminals during school time. Fourth, is the personal enrichment coming from enlarged social and vocational life. Tragically, social and vocational life is not enlarging for young people, who spend more and more time playing with their computers rather than with their peers. Their vocational life is becoming poorer because employers prefer to use complex software to enable them to use more low-paid and easily replaced unskilled workers. Worse, young people are being given the idea that they don't need to extend their general knowledge because they can find out anything they want to know from the Web.

These comments reflect the opinions of an earlier generation. Regardless of how well founded or how subjective they are, the underlying problem is that many young people are being inculcated with a sensual priority, and are not being given the possibility of knowing and preferring a richer, contemplative culture. The irony is that digital technology could be used in family life and formal schooling to give the average child the possibility of a far richer cultural life than all but the most gifted present-day children. The method is by using computers to develop basic skills.

\section{Automatic drill}

Advanced skills and accomplishments depend on the use of basic skills. The more basic skills that a person has, the more advanced their accomplishments can be. Basic skills are those skills that are applied without conscious thought. There was a time when skills such as reading, spelling, grammar and mental arithmetic were the normal result of elementary schooling. Nowadays, there are 
complaints at high bureaucratic levels that English spelling is too difficult for school children to learn; certainly there is plenty of evidence in public notices that they aren't learning it. What is the problem?

The problem is that basic skills come from persistent drill and practice. Ask any music teacher or athletics trainer. Fifty years ago, drill and practice was standard in primary schooling, even though it must have been very boring for the teachers. Today, it is dismissed with disgust by teachers as 'drill and kill'. The shame of this is that the digital computer can be vastly more effective at administering drill than any human primary classroom teacher. Automatic drill is intensely personal. The software can adapt the stored training to individual talents and capabilities, to individual moods and interests, and even to overcome defects such as dyslexia. It can be used to report to a teacher on each student's progress so that social classroom activities can be designed and corrective activities can be imposed.

Automatic drill is extremely versatile. With speech recognition it can be used with very young children. The customary primary skills of reading, writing and arithmetic can be developed way beyond present-day possibilities and, for quite young pupils, in different languages and writing systems. Less usual skills can be developed, such as singing, recitation, elocution, reading and composing music, recognising tunes and instruments, history, local and international geography, and many, many others. With haptic interfaces, like Nintendo's Wii for example, basic physical skills such as musical instrument performance and sign language could be developed.

Automatic drill is potentially revolutionary. In education, it would change the rôle of the teacher from autocrat to helpmate, eliminating fixed classes (every student would develop their own spectrum of individual skills) and making traditional tests and periodic examinations pointless (the software continually records basic skill levels, and advanced skills would be encouraged differently for different students). Outside the education system, it would empower people to develop basic skills personally and swiftly, once they identified their need and prepared to commit their time to drill. The introduction of pervasive automatic drill into schools would, of course, need to be done in stages. But the difficulty is to get it done at all.

Teachers have been thoroughly taught that drill is inhuman and, in any case, they would need themselves to be re-educated and trained to best exploit this technology. Politicians and demagogues would need to be convinced, or bypassed, to generate acceptance of a change in approach to education. Nevertheless, it is ethically imperative that the computing profession takes the lead in getting automatic drill accepted and adopted. This not to impose a particular culture on society but to give people in our society the potential to 
extend themselves in whatever culture they choose to adopt. It is also imperative because it will extend the capability of society in almost every way, an extension that is increasingly important because of the threats and challenges of a global nature that are becoming evident.

\section{Data standard}

While there is a general ethical imperative to get automatic drill widely accepted and practised, there is a customary danger to be avoided. In the past, new technologies such as radio and television have been seen as of enormous potential in improving education and community standards. As it happens, the educational and community potential has been swamped by commercial and marketing needs and influence. The same danger looms over automatic drill. Videogames, often of a murderous flavour, dominate the computing field at present, even at the level of chip manufacture. While these sensually absorbing computer applications are spreading ever wider, some commercial interests see some profit to be made from adapting these to mind-training use. This threatens the same fate for educational automatic drill as radio and television overwhelming use for marketing.

Software for automatic drill does, of course, need to be developed commercially. The computing profession, however, needs to press for this development to be versatile enough for teachers and parents to be able to develop and adapt their own drill packages, rather than being forced to accept whatever commercial enterprises see as most beneficial to their profits. The only way to do this is to establish, and to compel acceptance of, an international standard for the representation of the data used by the drill packages. This would mean that commercial interests, and open-source software developers, would be forced to develop software to best use the drill data that is being generated free from dependence on particular software packages.

The standard needs to define formats for two different categories of data: the subject matter data and the individual learner's data. These would necessarily be linked, but the subject matter data of a drill package would be general in nature and be incrementally improved, while the learner data would be generated through the use of the drill package by each learner.

\section{Conclusion}

The computing profession has some very important ethical responsibilities in our present day society. The first is to get binary digital technology seen as a technology that people use, and for which use people must bear the 
responsibility. Computing professionals should see themselves as bearing a special responsibility, and as needing to see that the public at large understands that technology itself is not responsible for problems caused by shortcomings of digital technology.

The second is to get the computing profession respected as a learned profession that is capable of improving the use of computers in society. This cannot be done now because, given that computers are so widely used, a clear and reasonable public image of the profession no longer exists. Such an image depends on the profession revising its scope and responsibilities.

The third is to see that the social benefits of binary digital technology are broadened and amplified, rather than the commercial and political benefits. The most important way to achieve this is to get the technology used in early education to increase the potential of students, rather than to make it cheaper for government and less demanding for teachers.

\section{References}

Holmes, WN, 2006, Computers and People, Wiley-IEEE Computer Society Press, Hoboken, New Jersey (ISBN-13: 978-0-470-00859-1, xxii+324pp).

Simpson, JA, Weiner, ESC (edd.), 1991, The Compact Oxford English Dictionary, Second Edition, Clarendon Press, Oxford (ISBN: 0-19-861258-3, xvii+2386pp).

Tootill, GC (ch.), 1968, IFIP-ICC Vocabulary of Information Processing, NorthHolland Publishing Company, Amsterdam (xii+2088pp). 